\title{
Role of sol-gel networking and fluorine doping in the silica Urbach energy
}

\author{
R. Lorenzi ${ }^{a}$, S. Brovelli ${ }^{c}$, F. Meinardi ${ }^{a, b}$, A. Lauria $^{a}$, \\ N. Chiodini ${ }^{a}$, A. Paleari ${ }^{\mathrm{a}, \mathrm{b}}$, \\ ${ }^{a}$ Department of Materials Science, University of Milano-Bicocca, Via R. Cozzi 53, \\ I-20125 Milano, Italy \\ ${ }^{\mathrm{b}}$ Consorzio Nazionale Interuniversitario per la Struttura della Materia (CNISM), \\ Italy \\ ${ }^{\mathrm{c}}$ Chemistry Division, Los Alamos National Laboratory, Los Alamos, New Mexico \\ 87545, USA
}

\begin{abstract}
We present the results of the analysis of the ultraviolet (UV) absorption edge of fluorine-modified sol-gel silica. UV transmission data, obtained by means of synchrotron radiation, have been analyzed in the spectral range $7.5-8.5 \mathrm{eV}$, with a spectral resolution of about $10 \mathrm{meV}$. Data on silica samples with different $\mathrm{F}$ content (from 0 to few $10^{-1} \mathrm{~mol} \%$ ) have been analyzed and compared with literature data on quartz and pure synthetic commercial silica. The analysis allows us to discriminate between the effects of the fluorine addition and those ascribable to structural peculiarities of the sol-gel networking. The estimated Urbach energy $E_{U}(T=0)$ ranges between 45 and $55 \mathrm{meV}$, higher that in crystalline quartz and lower than in commercial synthetic silica. The study of the temperature dependence of $E_{U}(T)$ shows that the fluorine modification of the silica network causes the lowering of the static disorder and the widening of the energy gap. However, there is also a relevant effect of the production process, since sol-gel silica samples show lower $E_{U}$ values with respect to other types of silica, quite independently of the fluorine content. The analysis of the Raman spectra however shows that the starting amount of fluorine-modified molecular precursor influences the network condensation process, independently of the final fluorine content into the matrix.
\end{abstract}

Key words: silica, sol-gel, fluorine, ultraviolet absorption spectroscopy, Raman Scattering 


\section{INTRODUCTION}

Glassy materials with high transparency in the ultraviolet (UV) and vacuum ultraviolet (VUV) region of the electromagnetic spectrum are of primal importance for laser technology, optical components, spectroscopy and semiconductors industry. In particular, $\mathrm{F}_{2}$-laser-based optical lithography requires glass photomasks with high radiation hardness and optical transmittance at the operation wavelength of $157 \mathrm{~nm}(7.9 \mathrm{eV})$ [1]. Pure, stoichiometric, and defect free $\mathrm{a}-\mathrm{SiO}_{2}$ is a key material for this application, mainly for the low thermal expansion coefficient. However, fluorine doping may greatly improve the performance, thanks to the fluorine effect that shifts the absorption edge towards slightly higher energy [2-6]. The reason of this effect may be found in several concurrent mechanisms, related to the greater strength of the $\mathrm{Si}-\mathrm{F}$ bond with respect to the Si-O bond, the quenching of UV absorbing point defects, the structural relaxation of the matrix caused by the reduction of strained bonds, and the lowering of glass density [6]. Furthermore, the optical transparency in the absorption edge is also related to the vibrational density of state that influences the thermal dependence of the Urbach tail. The vibrational density of states is in turn related to the material networking, which is determined by the specific thermal history and fabrication technique. In fact, the absorption edge in insulators and semiconductors in the so-called Urbach region shows a temperature dependence following an exponential law described by

$$
\alpha(E)=\alpha_{0} \exp \left[\frac{E-E_{0}}{E_{U}(T)}\right]
$$

where $\alpha$ and $E$ are the absorption coefficient and the energy of the incident photon, respectively, while $E_{0}$ and $E_{U}$ are parameters that characterize the absorption edge. Since $E_{U}(T)$ is a function of the temperature, Eq. 1 represents a bundle of straight lines in semilog plot, converging in the point $\left[E_{0}, \ln \left(\alpha_{0}\right)\right]$ called Urbach focus. The inverse of their slopes is proportional to the Urbach energy $E_{U}(T)$, thus representing the broadness of the absorption tail. This energy is proportional to the internal energy and it depends on both dynamic (phononic) and static (structural) disorder [7]. More specifically, dynamic disorder arises from the thermal occupancy of the phonon states, whereas the temperature independent static disorder is a measure of the ratio between the effective Urbach energy at $T=0 \mathrm{~K}$ and the zero-point internal energy for the ideal case. Indeed, the Urbach energy may be expressed as the sum of two independent terms, one referred to the dynamic disorder $\left(E_{d y n}(T)\right)$ and the other one to the static disorder $\left(E_{\text {stat }}=X \hbar \omega_{0} / 2 \sigma_{0}\right)$ :

$$
E_{U}(T)=E_{\text {stat }}+E_{\text {dyn }}=\frac{\hbar \omega_{0}}{\sigma_{0}}\left[\frac{1+X}{2}+\frac{1}{\exp \left(\hbar \omega_{0} / k_{B} T\right)-1}\right]
$$


where $\hbar \omega_{0} / 2$ is the zero-point energy of the quantum oscillator in the Einstein model of a solid, $X$ an adimensional parameter of structural disorder, and $\sigma_{0}$ the Urbach edge parameter defined by the constraint $\lim _{T \rightarrow \infty} E_{U}(T)=$ $k_{B} T / \sigma_{0}$. As far as the study of the Urbach energy as a function of the temperature may give relevant information about the phonon population and the intrinsic disorder, this parameter is not directly related to the electronic structure, which instead determines the energy position of the Urbach tail itself. Since the concept of "optical band gap" $E_{G}$ is roughly applicable to insulators

and the absorption coefficient $\sqrt{\alpha(E, T) E} \propto\left[E-E_{G}(T)\right]$ in the Tauc region is hardly observable in silica-based materials, other data representations are preferred for the evaluation of the energy of the band to band transitions, such as isoabsorption or isoenergetic curves.

In the present work we report the analysis of the absorption edge and the dynamic and static contributions to the Urbach tail in different fluorine modified and pure silica samples produced by sol-gel method, compared with commercial synthetic silica. We show that the peculiar networking arising from the sol-gel synthesis plays an important role in determining the absorption edge, also in fluorine modified silica.

\section{EXPERIMENTAL PROCEDURE}

\subsection{Samples}

Optical grade samples have been prepared by the sol gel technique with tetraethyl orthosilicate $\left(\mathrm{Si}\left(\mathrm{OCH}_{2} \mathrm{CH}_{3}\right)_{4}, 99.999 \%\right)$ as silicon precursor and different initial concentrations of triethoxyfluorosilane $\left(\mathrm{SiF}\left(\mathrm{OCH}_{2} \mathrm{CH}_{3}\right)_{3}, 95\right.$ $\%)$ as fluorine precursor and using pure ethanol as a solvent. Gelation occurs in a few hours after addition of pure water. Molar ratios 1:7.4:11.5 of $\mathrm{Si}: \mathrm{H}_{2} \mathrm{O}: \mathrm{EtOH}$ was adopted. Different thermal treatments were carried out on the xerogels so as to obtain different structural features and effects of the fluorine modifier as dehydrating agent within the sample set. Samples were sintered in pure oxygen flux with a rate temperature of 2,4 or $6{ }^{\circ} \mathrm{C} / \mathrm{h}$ to reach a $10 h$ final stasis temperature at 1100 or $1200{ }^{\circ} \mathrm{C}$. Samples were prepared with four different initial fluorine contents $(2,5,8$ and $10 \% \mathrm{~mol} \mathrm{~F} / \mathrm{mol} \mathrm{Si})$ and different thermal treatments. Synthesis parameters are summarized in Tab. 1. Optical grade glasses in form of disks of about $1 \mathrm{~cm}$ in diameter with plane and parallel surfaces have obtained, with final thicknesses ranging from 180 to $530 \mu \mathrm{m}$, with deviations from parallelism less than $1 \mu \mathrm{m}$ over the entire area. 


\subsection{Measurements}

The structural features have been investigated by Raman spectroscopy in backscattering configuration, with a spectral resolution of $1 \mathrm{~cm}^{-1}$ and using a $633 \mathrm{~nm}$ He-Ne laser line as light source. Hydroxyl content was calculated from the amplitude of the infrared (IR) silanol stretching absorption band at 3670 $\mathrm{cm}^{-1}$ [8] detected by means of a $\mu$-FTIR spectrometer with a resolution of 2 $\mathrm{cm}^{-1}$. The residual fluorine content was inferred from Raman and refractive index measurements comparing our results with F-modified silica with known $\mathrm{F}$ content $[3,8]$, collecting the refractive index value by using a prism coupler apparatus at $633 \mathrm{~nm}$ with an uncertainty of $10^{-4}$. VUV absorption spectra, in the temperature range $25-300 \mathrm{~K}$, were collected by means of synchrotron radiation at the SUPERLUMI experimental station of HASYLAB (DESY, Hamburg) with a spectral resolution of $0.2 \mathrm{~nm}$. The absorption coefficient $\alpha$ was derived measuring the light beam intensity transmitted by the sample and assuming $I_{c}=\left(I_{0}-I_{s}\right)(1-R)^{2} \exp [-\alpha / L]$, where $I_{0}$ is the beam intensity without sample, $I_{c}$ the collected intensity, $\mathrm{L}$ is the sample thickness, $(1-R)^{2}$ and $I_{s}$ the reflection and diffusion corrections, respectively. The scattered intensity $I_{s}$ has been assumed to be the signal recorded at wavelengths with negligible transmittance. Reflectivity at normal incidence $R(\lambda)=(1-n(\lambda))^{2} /(1+n(\lambda))^{2}$ has been calculated from a Sellmeier relation derived from dispersive measurement on similar F-modified silica glasses $[4,8]$ in order to obtain values of refractive index at $633 \mathrm{~nm}$ consistent with those we have experimentally observed. Error on $\alpha$ deriving from the uncertainty on $(1-R)^{2}$ in the Urbach region, from 8 to $8.4 \mathrm{eV}$, is less than $2 \%$.

\section{RESULTS}

Fig. 1 shows a representative Raman spectrum of a fully densified, fluorine modified, sol-gel silica glass. The peak at $945 \mathrm{~cm}^{-1}$ is due to the Si-F stretching mode, whereas the spectral features at 440 (broad peak), 490 (sharp peak), $606,800,1050$, and $1160 \mathrm{~cm}^{-1}$ are ascribable to the silica matrix and correspond to the $\omega_{1}, D_{1}, D_{2}, \omega_{3}, \omega_{4}(\mathrm{TO})$, and $\omega_{4}(\mathrm{LO})$ modes, respectively. The $\omega_{n}$ features arise from the band limits of the intrinsic phonon band structure of the tetrahedrally coordinated amorphous network of silicon dioxide [9], whereas $D_{1}$ and $D_{2}$ are attributed to the breathing modes of four and three membered planar rings of $\mathrm{SiO}_{4}$ tetrahedra, respectively $[10,11]$. After baseline correction, Raman spectra were normalized with respect to the amplitude of the $\omega_{3}$ mode, which is proportional to the total amount of $\mathrm{Si}-\mathrm{O}-\mathrm{Si}$ bond, irrespective of the OH-group or other impurities content, as well as of the matrix porosity [12].

Table 1 lists the data related to the initial and residual fluorine content to- 
gether with additional data on the thermal treatment conditions and the hydroxyl concentration. It is worth noting that the amount of residual fluorine is almost independent of the initial F-precursor in the sol-gel. On the contrary, the sintering heating rate plays a relevant role on the halogen retention. Sample F50 is a neat example of such an effect. In fact it shows the highest fluorine retention, although it has the lowest starting doping. This result was obtained thanks to a fast sintering rate which modified the kinetic equilibrium between pore collapse and fluorine release with respect to the other samples.

Fig. 2 shows the corrected absorption spectra at room temperature of the samples under study. The improvement in the VUV transmittance from F0 (full line) to F50 (dotted line) confirms the fluorine role as a band gap widening agent. The observed effects are comparable to those reported for fluorinated silica prepared with other techniques [6]. The inset in Fig. 2 shows the temperature evolution of the absorption spectrum in the most fluorinated sample (F50). Lowering the temperature from 300 down to $25 \mathrm{~K}$ causes a shift of about $0.15 \mathrm{eV}$ toward higher energy in the absorption tail.

Thermal effects on the energy shift are represented in Fig. 3 as isoabsorption curves, where the photon energy at a fixed value of the absorption coefficient $\left(\alpha=200 \mathrm{~cm}^{-1}\right)$ is plotted as a function of the temperature. We observe an increment by decreasing the temperature, reaching a plateau for temperature lower than $\approx 75 \mathrm{~K}$. We note once more that the sample F0 is the most absorbing glass within our set and its absorption properties are comparable with solgel silica produced from a different process but containing similar hydroxyl content [16]. On the other hand, the highest optical transmittance is observed in F28 in the low temperature range whereas, at $T>80 \mathrm{~K}$, F50 becomes the sample with the lowest optical losses, according to the room temperature behavior reported in Fig. 2. For sake of clarity, the shift of isoabsorption photon energies is plotted in the inset of Fig. 3 referred to the sample F50. Two distinct kinds of temperature dependence are evidenced: i) samples F26 and F50 show a relatively small dependence of about $3 \mathrm{meV}$ over the entire temperature range, ii) samples F28 and F0 show a more pronounced temperature dependence, the isoabsorption energy range being about $20 \%$ wider than in the other two samples.

Fig. 4 shows the Urbach tail analysis within the sample set. Pure amorphous and crystalline quartz are also plotted for comparison. Urbach energies $E_{U}$ have been derived fitting the absorption spectra by means of Eq. 1 and minimizing the data dispersion in the Urbach focus $\left(E_{0}, \ln \left(\alpha_{0}\right)\right)$. The region of linearity in semi-log scale ranges over about 1.5 order of magnitude in $\alpha$, up to 500 or $600 \mathrm{~cm}^{-1}$ depending on the glass thickness. The solid lines are the results of fitting the data by means of Eq. 2. The best-fit parameter values are reported in Tab. 2 with an uncertainty of about $5 \%$. The limit value of the Urbach energy for $T \rightarrow 0$ corresponds to the quantum limit of 
$E_{U}(0)=\hbar \omega_{0}(1+X) / 2 \sigma_{0}$. In our samples this value is comprised between the two limiting cases of pure silica $(70 \mathrm{meV})$ and quartz $(36 \mathrm{meV})[13,14]$, except for the non-fluorinated sample $\mathrm{F} 0$ that shows a low temperature limit value of $93 \mathrm{meV}$. The zero point quantum energy of the oscillator determines also the temperature at which the Urbach energy reaches the limit value $E_{U}(0)$. In fact $\hbar \omega_{0} / k_{B}$ is the Debye temperature $\Theta_{D}$ of the system. The latter may be regarded as a measure of the temperature above which all modes are excited or, equivalently, an indicator of the minimum thermal energy needed to populate the excited state. Thus, for an ideal Einstein solid, greater values of $E_{U}(0)$ correspond to higher temperatures at which the knee in $E_{U}(T)$ occurs. This behavior is valid for quartz and pure silica, where the plateau is reached for $T \approx 110 \mathrm{~K}$ and $\approx 200 \mathrm{~K}$, respectively, and the value of $E_{U}(0)$ increases by a factor 2 passing from crystalline to amorphous structure. On the other hand, in our samples, $E_{U}(T)$ reaches the quantum limit at $T \approx 100 \mathrm{~K}$ regardless either the measured $E_{U}(0)$ or the residual fluorine content, in agreement with the resulting $\hbar \omega_{0}$ value, which is only slightly dispersed around a mean value of $36.5 \mathrm{meV}$ within the sample set.

\section{DISCUSSION}

The collected data evidence clear differences within the investigated set of samples, as well as clear peculiarities with respect to the silica and quartz references $[13,14]$. These differences are mainly related to the widening of the band gap and to the sharpening of the absorption edge with respect to commercial silica. We may note that gap-widening is just the expected effect of fluorine-modification of the electronic structure of the whole system. However, the detailed analysis of the iso-absorption curves (inset of Fig. 3) shows small differences among the investigated samples that suggest also indirect effects related to the fluorine modification of $\mathrm{OH}$-content and networking degree. In fact, minor contributions to the gap widening with respect to commercial silica should arise as a result of the peculiar sol-gel networking, since the low structural stress with respect to material produced from quenching processes should lead to slightly wider energy gap [15]. Furthermore, another concurrent effect contributing to the apparent gap widening, is the fluorine-induced decrease of the $\mathrm{OH}$-groups content which may give to non negligible absorption at around $8 \mathrm{eV}$. This effect may be observed in the changes of isoabsorption energy in Fig. 3, where no subtraction of hydroxyl spectral contributions has been carried out, at variance with other studies on pure dried sol-gel silica [17]. As a result, looking at the representation reported in inset of Fig. 3, additional absorption contributions are identified, thanks to their different thermal behavior, in samples with higher $\mathrm{OH}$ content (F28 and F0) taking as a reference the less hydrated sample (F50). Furthermore, a role in the gap 
widening effect is also played by the structural disorder, either static or dynamic, arising from the specific phonon spectrum of the material. In fact, further modifications with respect to the reference materials appear within the investigated set of samples, concerning the steepness of the absorption edge and its thermal dependence evidenced by the parameters $\hbar \omega_{0}, \sigma_{0}$ and $X$ (Tab. 2), which reflect the electron-phonon coupling and the static structural disorder. Specifically, looking at Tab. 2, we observe that $\hbar \omega_{0}$ is particularly low and it is independent of the fluorine content. This fact evidences a peculiarity probably related to the effects of the production method on the average structure of the silica network. The contribution $X$ to the Urbach energy is instead tendentially lower in fluorinated samples within the investigated set (except for F28 whose value is probably significantly affected by OH-related absorption at low temperature), whereas is much higher compared with the reference materials. This result supports the already suggested role of fluorine in reducing the static disorder of the silica structure, even though it evidences a much wider spreading of structural parameters than in fused silica and, of course, than in quartz. To analyze these differences in more detail, we have to consider the effects of the synthesis condition on the structural disorder of our systems. The Raman analysis shows that fluorine doping modifies not only the electronic properties of the investigated glass, but also the vibrational density of state (VDOS) of the system. Specifically, we observe a reduction of the amplitude of the $D_{2}$ mode as a function of the residual fluorine content, confirming that high halogen concentrations reduce the amount of strained bonds [6]. It is worth noting that, independently of the final fluorine content, the fluorine addition plays also a role during the synthesis in determining the matrix condensation and the final structural features. In fact, in samples F26 and F28, the difference in the $D_{2}$ mode intensity can be attributed exclusively to the different initial fluorine content and its effect on the F-modified solgel synthesis and condensation of the amorphous silica structure, because the final materials indeed show very similar fluorine contents, after identical sintering conditions. Although the possible chemical reactions between the silica matrix and the fluorine precursor are not yet clear, we may argue that the thermal treatment activates F-derivatives responsible for dehydroxylation and structural matrix alterations. In sample F0, for instance, even though the $\mathrm{SiF}$ stretching mode is not detectable, indication of some effects ascribable to fluorine precursors in the sol can be recognized in the low hydroxyl content and in the less stressed structure. As a matter of fact, for pure sol-gel silica prepared by the same route of F-modified materials - but without F-precursors - the normalized $\mathrm{D}_{2}$ mode amplitude is about $16 \%$ higher and $\mathrm{OH}$ content is one order of magnitude greater than in sample F0 [18]. Analogous behavior occurs for samples F26 and F28, where the fluorine molarity in the sol influences the final hydroxyl amount and the intensity of the $D_{2}$ peak, in spite of their similar residual $\mathrm{F}$ contents. 


\section{CONCLUSIONS}

In the present work we have shown that the analysis of the temperature dependence of the Urbach tail clarifies the real role of fluorine in modified silica from sol-gel. In fact, whereas the fluorine effect on $E_{U}(300 \mathrm{~K})$ has been already remarked in other studies, our work clarifies that the main contribution ascribable to fluorine is the band gap widening and the lowering of the static structural disorder. On the other hand, other features potentially interesting for optimizing the VUV transmittance of fluorine modified silica are strongly related to the production method. Specifically, the steepness of the absorption edge is a feature essentially dependent on the peculiar structural networking determined by the sol-gel synthesis, which finally causes the lowering of $E_{U}$.

\section{Acknowledgment}

The research leading to these results has received funding from the European Community's Seventh Framework Programme (FP7/2007-2013) under grant agreement n 226716. 


\section{References}

[1] K. Ronse, Compt. Rend. Phys., 7 (2006) 844.

[2] M. Mizuguchi, L. Skuja, H. Hosono, T. Ogawa, J. Vac. Sci. Technol. B 17 (1999) 3280 .

[3] C. M. Smith, L. A. Moore, J. Fluorine Chem. 122 (2004) 81.

[4] K. Tsukuma, N. Yamada, S. Kondo, K. Honda, H. Segawa, J. Non-Cryst. Sol. 127 (1991) 191.

[5] A. Paleari, F. Meinardi, A. Lauria, R. Lorenzi, N. Chiodini, S. Brovelli, Appl. Phys. Lett. 91 (2007) 141913.

[6] L. Skuja, K. Kajihara, Y. Ikuta, M. Hirano, H. Hosono, J. Non-Cryst. Sol. 345-346 (2004) 328.

[7] G. D. Cody, T. Tiedje, B. Abeles, B. Brooks, Y. Goldstein, Phys. Rev. Lett. 47 (1981) 1480.

[8] K. M. Davis, A. Agarwal, M. Tomozawa, K. Hirao, J. Non-Cryst. Sol. 203 (1996) 27.

[9] F. L. Galeener, Phys. Rev. B 19 (1979) 4292.

[10] F.L. Galeener, Solid State Commun. 44 (1982) 1037.

[11] A. Pasquarello, R. Car, Phys. Rev. Lett. 80 (1998) 5145.

[12] F. L. Galeener, G. Lucovsky, Phys. Rev. Lett. 37 (1976) 1474.

[13] I. T. Godmanis, A. N. Trukhin, K. Hübner, Phys. St. Sol b 116 (1983) 279.

[14] K. Saito and A. Ikushima, Phys. Rev. B 62 (2000) 8584.

[15] L. Martin-Samos, G. Bussi, A. Ruini, E. Molinari, M. J. Calda, Phys. Rev. B 81 (2010) 081202.

[16] E. Vella, R. Boscaino, Phys. Rev. B 79 (2009) 085204.

[17] E. Vella, R. Boscaino, G. Navarra, Phys. Rev. B 77 (2008) 165203.

[18] A. Lauria, PhD Thesis, University of Milano-Bicocca (Milano, Italy, 2008). 
Table 1

Starting concentration $[\mathrm{F}]_{0}$ of fluorine precursor (mol\%), heating rate $k\left({ }^{\circ} \mathrm{C} / h\right)$, final sintering temperature $T_{\text {sint }}\left({ }^{\circ} \mathrm{C}\right)$, final F relative content $[\mathrm{F}]$ estimated from Raman and refractive index data (maximum normalized to 100), and hydroxyl group content $[\mathrm{OH}]$ from IR spectra $(\mathrm{ppm})$. Uncertainty is about $10 \%$ except for $[\mathrm{F}]_{0}(0.01 \%)$. The order of magnitude of the absolute value of final fluorine content is estimated $10^{3} \mathrm{ppm}$ for $[\mathrm{F}]=100,[5,6]$.

\begin{tabular}{lccccc}
\hline sample & {$[\mathrm{F}]_{0}$} & $k$ & $\mathrm{~T}_{\text {sint }}$ & {$[\mathrm{F}]$} & {$[\mathrm{OH}]$} \\
\hline F28 $(\triangle)$ & 10 & 6 & 1100 & 56 & 28 \\
F26 (•) & 8 & 6 & 1100 & 52 & 17 \\
F0 ( $)$ & 5 & 2 & 1200 & $<1$ & 180 \\
F50 (०) & 2 & 10 & 1100 & 100 & 4 \\
\hline
\end{tabular}

Table 2

Parameters $\hbar \omega_{0}, \sigma_{0}, X$, and Urbach focus coordinate $\left(E_{0}, \alpha_{0}\right)$ from the fit of the Urbach tail of the absorption edge according to Eqs. 1 and 2. Sample-labels are the same of Tab. 1. Quartz and silica values are calculated from data in [13] and [14], respectively, with an uncertainty of about $10 \%$.

\begin{tabular}{ccccccc}
\hline & $\mathrm{F} 28$ & $\mathrm{~F} 26$ & $\mathrm{~F} 0$ & $\mathrm{~F} 50$ & silica & quartz \\
\hline$\hbar \omega_{0}(\mathrm{meV})$ & $37 \pm 2$ & $38 \pm 2$ & $35 \pm 2$ & $36 \pm 2$ & 79 & 46 \\
$\sigma_{0}$ & $1.0 \pm 0.05$ & $0.72 \pm 0.04$ & $0.81 \pm 0.04$ & $0.76 \pm 0.04$ & 0.66 & 0.63 \\
$X$ & $2.1 \pm 0.1$ & $0.73 \pm 0.04$ & $2.6 \pm 0.1$ & $0.98 \pm 0.05$ & 0.16 & $5 \times 10^{-5}$ \\
$E_{0}(\mathrm{eV})$ & 9.12 & 8.77 & 9.05 & 8.77 & - & 9.10 \\
$\alpha_{0}\left(\mathrm{~cm}^{-1}\right)$ & $10^{7.97}$ & $10^{5.85}$ & $10^{6.11}$ & $10^{5.88}$ & - & $10^{6.8}$ \\
\hline
\end{tabular}




\section{FIGURE CAPTIONS}

Figure 1 - Raman spectrum of fluorine-modified silica from sol-gel synthesis (sample F50). $D_{2}$ and Si-F modes are indicated, together with the intrinsic $\omega_{3}$ structure used to normalized the spectra of all samples. Inset: relation between the intensities of the $D_{2}$ and Si-F modes in the samples F28 $(\triangle)$, F26 $(\bullet)$, F0 $(\mathbf{\nabla})$, and F50 (o).

Figure 2 - Optical absorption edge of fluorine-modified sol-gel silica samples F28 (dashed-dotted line), F26 (dashed line), F0 (full line), and F50 (dotted line) at $300 \mathrm{~K}$. Inset: Temperature shift of the absorption edge in sample F50 from 300 to $10 \mathrm{~K}$ (step of $25 \mathrm{~K}$ ).

Figure 3 - Temperature dependence of isoabsorption energy - calculated as the energy at which the absorption spectrum takes the value of $200 \mathrm{~cm}^{-1}$ in the samples F28 $(\triangle)$, F26 $(\bullet), F 0(\mathbf{v})$, and F50 (o). Inset: differences of isoabsorption energy, referred to sample F50. Uncertainty is within the symbol size.

Figure 4 - Temperature dependence of the Urbach energy $E_{U}$ obtained by fitting the absorption edge with Eq. 1 in the samples F28 $(\triangle)$, F26 (•), F0 $(\boldsymbol{\nabla})$, and F50 (०). Curves are obtained by fitting the data with Eq. 2. Curves obtained by fitting literature data on commercial silica [14] and quartz [13] are also reported for comparison. 


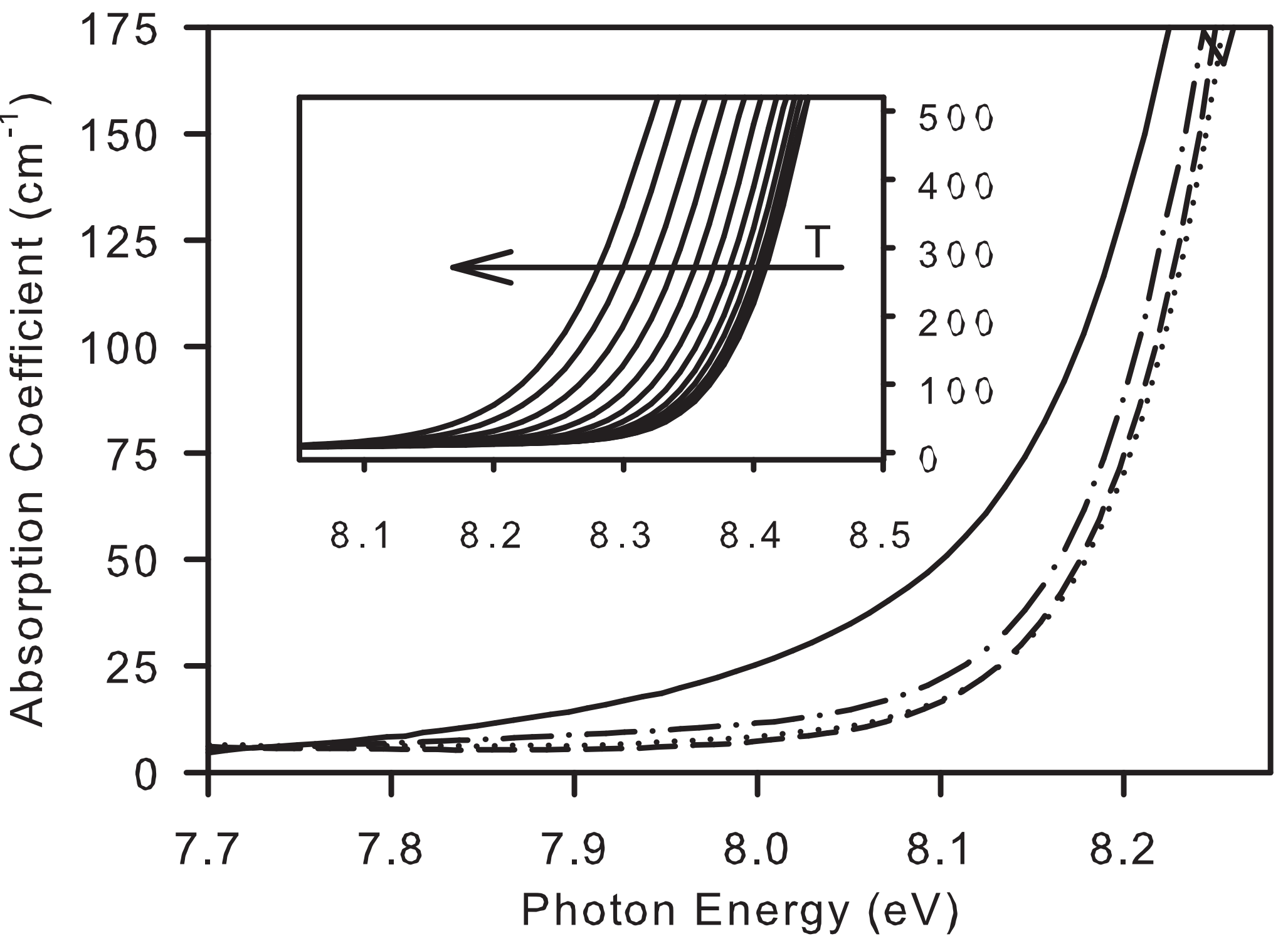




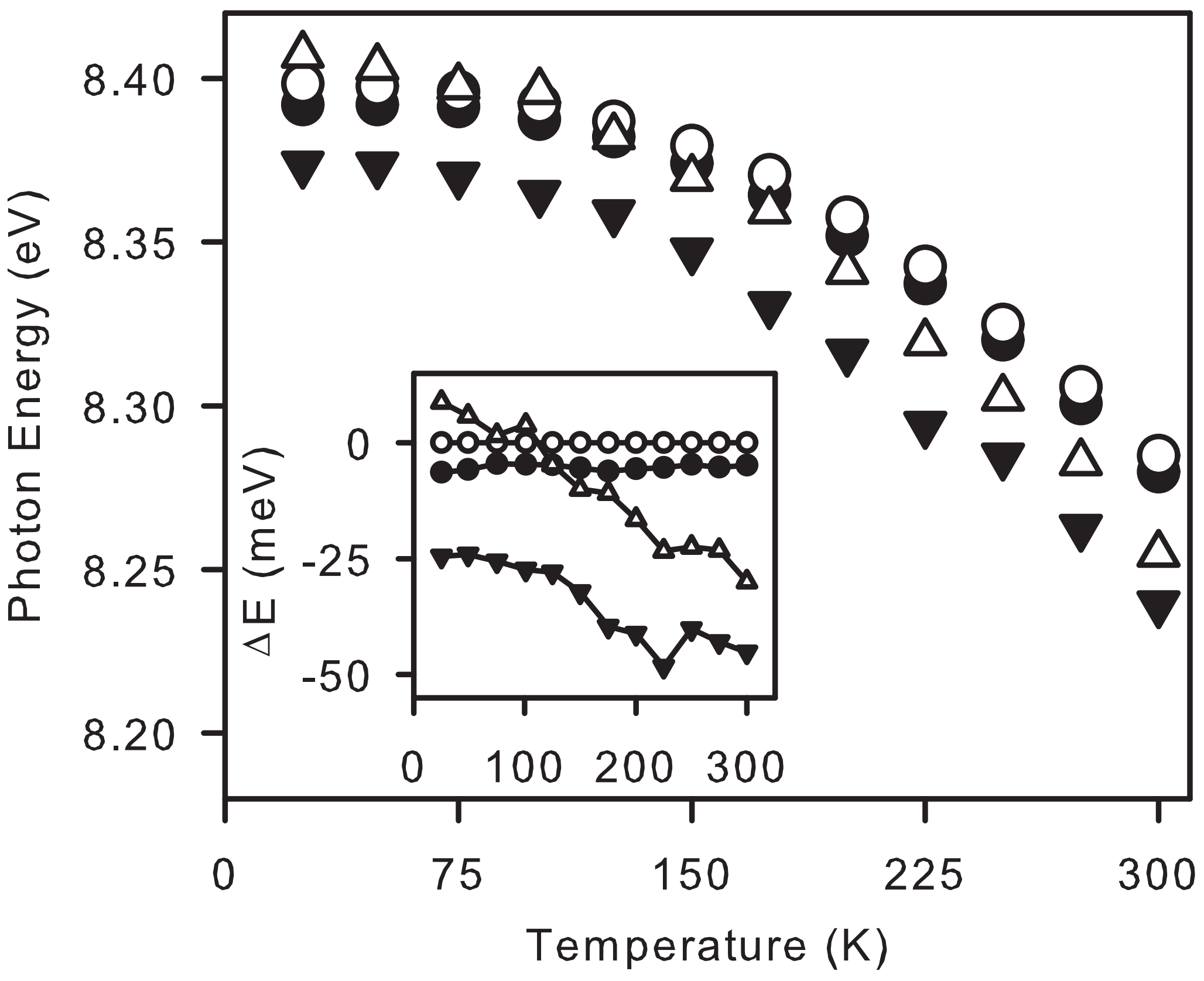




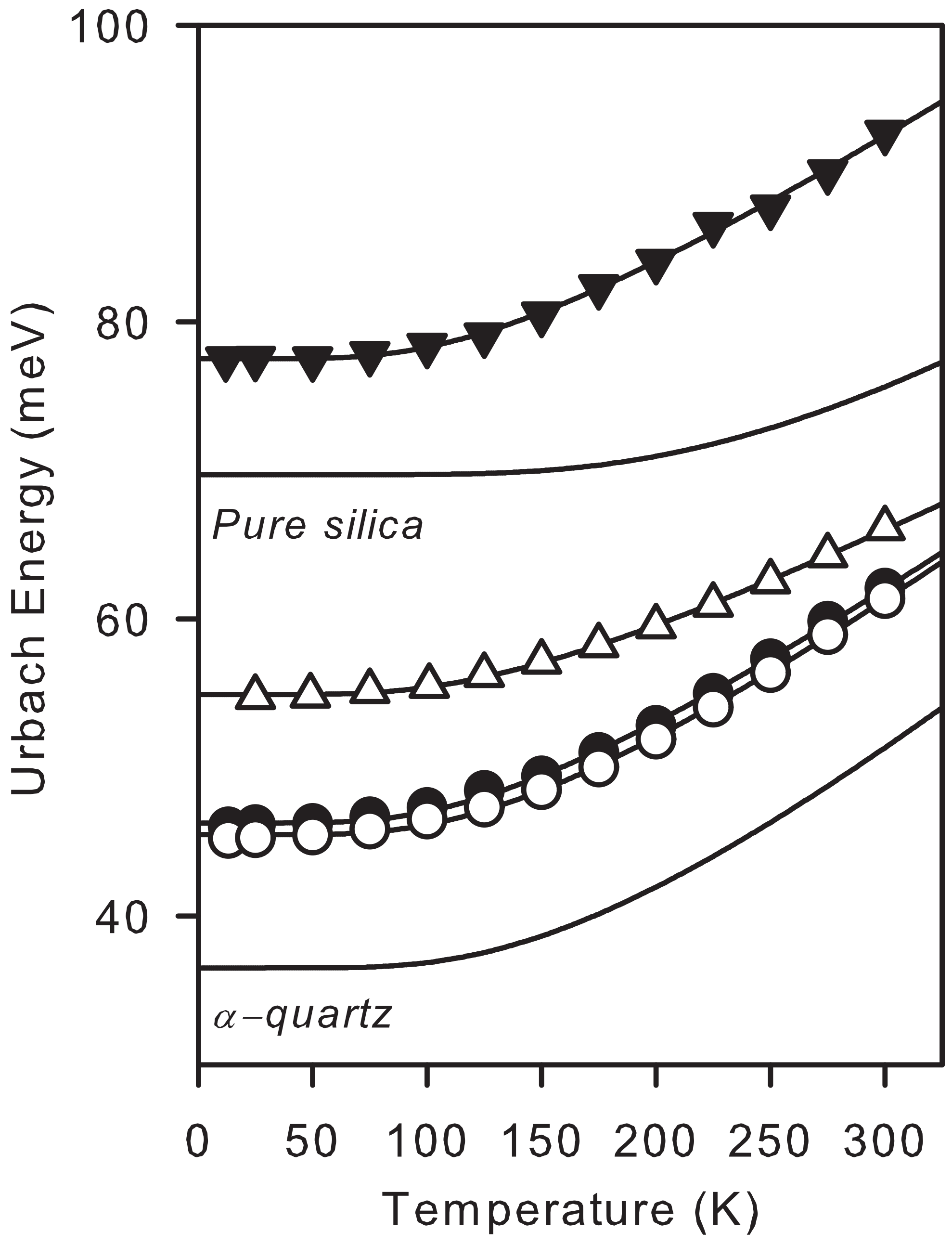

